\title{
Discriminative stimuli that follow a delay have added value for pigeons
}

\author{
KELLY A. DIGIAN, ANDREA M. FRIEDRICH, and THOMAS R. ZENTALL \\ University of Kentucky, Lexington, Kentucky
}

\begin{abstract}
Clement, Feltus, Kaiser, and Zentall (2000) reported that pigeons prefer discriminative stimuli that require greater effort (more pecks) to obtain over those that require less effort. In the present experiment, we examined two variables associated with this phenomenon. First, we asked whether delay of reinforcement, presumably a relatively aversive event similar to effort, would produce similar effects. Second, we asked whether the stimulus preference produced by a prior relatively aversive event depends on its anticipation. Anticipation of delay was accomplished by signaling its occurrence. Results indicated that delays can produce preferences similar to those produced by increased effort, but only if the delays are signaled.
\end{abstract}

Animals typically prefer stimuli that predict less rather than more effort and shorter rather than longer delays to reinforcement (Hull, 1943). However, if those stimuli follow more versus less effort or longer versus shorter delays, it is not obvious whether preferences should be found. More specifically, if differential effort is followed by a stimulus that signals reinforcement, will preference for the stimulus be affected by the effort required to obtain it?

On the one hand, stimulus preference should be affected by its consequents rather than by its antecedents (Hull, 1943), so preference may not be affected. On the other hand, if effort becomes part of the context in which the stimulus appears (Bouton, 1993) or if backward associations form between the reinforcement and the effort that precedes it (Spetch, Wilkie, \& Pinel, 1981), then antecedent effort may affect preference in the same way as consequent effort, and stimuli following low effort should be preferred. Finally, it is possible that the antecedent effort is contrasted with the conditioned reinforcing properties of the stimulus, thus enhancing the value of the stimulus.

Clement, Feltus, Kaiser, and Zentall (2000) investigated the effect of effort on pigeons' preference for a conditioned reinforcer that followed. At the start of each trial, a white light was presented on the center response key. On some trials, a single peck was sufficient to turn on a simultaneous discrimination on the side keys (e.g., red + , yellow - ). On the remaining trials, 20 pecks were required to turn on a different simultaneous discrimination on the side keys (e.g., green + , blue-). Following training, the pigeons were tested on probe trials involving a choice between the two $\mathrm{S}+$ stimuli. The results indicated that the pigeons preferred the $\mathrm{S}+$ that followed

This research was supported by National Institute of Mental Health Grant MH-59194. Correspondence concerning this article should be sent to T. R. Zentall, Department of Psychology, University of Kentucky, Lexington, KY 40506-0044 (e-mail: zentall@uky.edu). the greater effort over the $\mathrm{S}+$ that followed the lesser effort. Thus, prior effort did affect preference for the stimuli that followed, and the result can be described as a contrast effect - more specifically as contrast between the state of the organism at the end of the white-light response requirement and the state of the organism at the onset of the discriminative stimuli. In other words, assuming that the pigeon is in a relatively neutral state at the start of a trial and in a more negative state after having to peck 20 times, the appearance of the discriminative stimuli (signaling reinforcement) should represent a relatively large improvement in conditions. On low effort trials, however, there should be little opportunity to establish a negative state and the appearance of the discriminative stimuli should represent a smaller improvement in conditions. If contrast is responsible for this preference, it suggests that other differentially preferred events might be substituted for the differential peck requirement and should have similar effects on preference for the discriminative stimuli that follow. For example, if on some trials, an initial response immediately produced discriminative stimuli, whereas on other trials, an initial response produced the discriminative stimuli only after a delay, would a similar preference be found for the $\mathrm{S}+$ that in training followed the delay? The purpose of the present experiment was to test this counterintuitive hypothesis.

One potentially important difference between the manipulation of prior effort reported by Clement et al. (2000) and the manipulation of prior delay suggested here is that in the case of the effort manipulation, if the discriminative stimuli do not appear following the first peck, it is predictable that 19 additional pecks will be required to produce the stimuli. That is, the pigeon can anticipate that additional effort will be required. In the case of the delay manipulation, however, unless it is signaled, the relatively more aversive event (i.e., the delay) cannot be anticipated when responding is required. Thus, a secondary purpose of the present experiment was to determine the importance 
of anticipation of the relatively more aversive event. To examine the effect of anticipation of an aversive event, for one group of pigeons, the initial stimulus on the center key was made to be informative. That is, prior to a response, it signaled whether or not a delay would follow. For the other group of pigeons, the initial stimulus did not signal whether or not a delay would follow. If contrast is involved, the preference for the stimuli that follow the delay may be more pronounced in the signaled group because the delay can be expected from the start of the trial.

\section{METHOD}

\section{Subjects}

Subjects were 16 White Carneau pigeons (Columba livia) of unknown sex that were purchased from the Palmetto Pigeon Plant (Sumter, SC). The pigeons had had previous experience with conditional color and shape discriminations. The pigeons were singly housed in a colony room on a 12:12-h light:dark cycle. The pigeons were food deprived to stabilize their weight at $80 \%$ of their freefeeding weight throughout the experiment, but they had free access to water and grit in their home cages.

\section{Apparatus}

The experiment was conducted in an operant chamber (BRS/LVE, Laurel, MD), which was controlled by a microcomputer in a sepa- rate room. The chamber could be illuminated by a houselight located at the top center of the response panel. There were three response keys mounted in a horizontal line on the response panel. Each key was located directly in front of a projector (Industrial Electronics Engineers, Model 10, Van Nuys, CA) that was capable of projecting stimuli onto the key (white and a vertical or a horizontal white line on a black background on the center key, and four hues - red, yellow, green, and blue — on the side keys). A feeder (illuminated by a feeder light) located below the center key provided reinforcement (1.5-sec access to mixed grain).

\section{Procedure}

Training. For the pigeons in the unsignaled group $(n=8)$, trials were initiated by a white initial stimulus presented on the center key that required 20 pecks and was followed by a pair of discriminative stimuli presented on the left- and right-side keys. On half of the 48 trials in each training session, the discriminative stimuli followed the 20 pecks immediately. On the remaining trials, the 20 -peck response requirement was followed by a 6 -sec dark delay prior to presentation of a different pair of discriminative stimuli. The pair of hues that followed the delay and the hue designated as the $\mathrm{S}+$ were counterbalanced over birds. One peck to the $S+$ stimulus resulted in reinforcement. Trials were separated by a $10-\mathrm{sec}$ intertrial interval, during which the houselight was illuminated. The design for the unsignaled group is presented in the top panel of Figure 1.

For pigeons in the signaled group, half of the trials were initiated by a vertical-line stimulus and the remaining trials were initiated by a horizontal-line stimulus. On vertical-line trials, the discrimi-
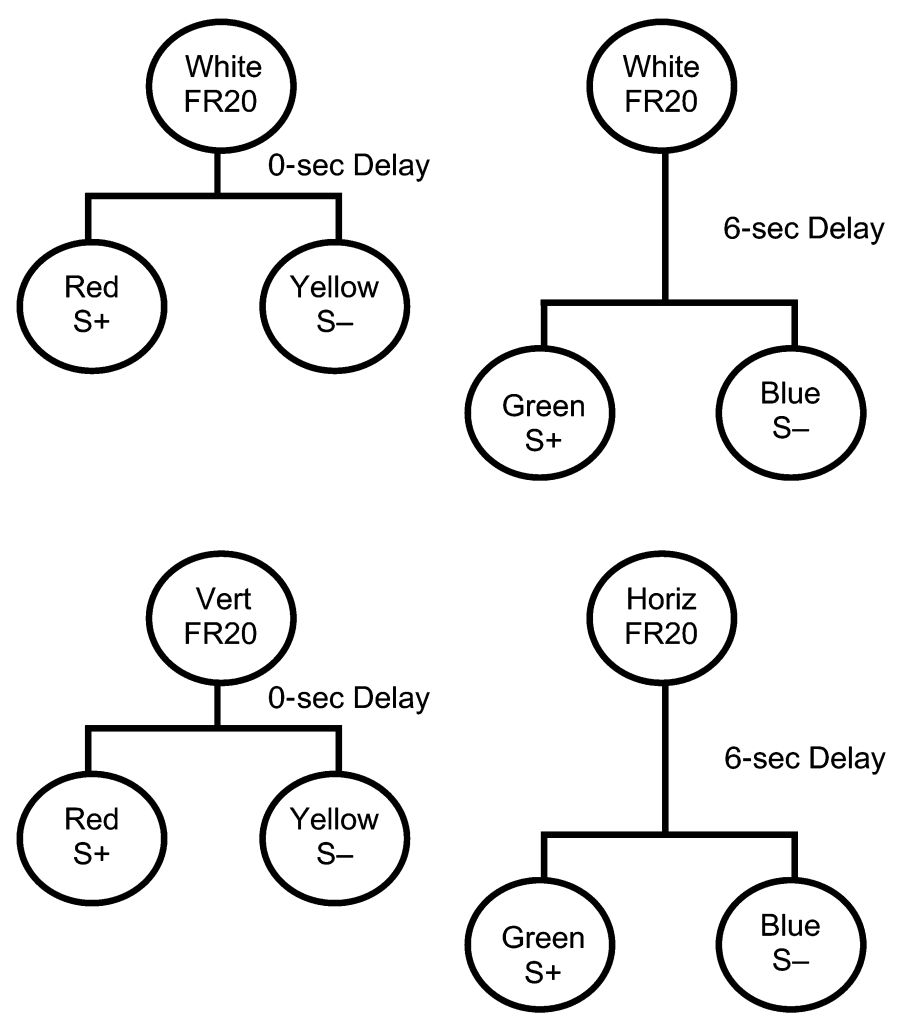

Figure 1. Sequence of events experienced by pigeons in the unsignaled group (top panel) and the signaled group (bottom panel) during training. FR20 = twenty pecks were required. The colors that appear are merely illustrative. In fact, the colors that served as the positive and negative stimuli for delay and no-delay trials were counterbalanced over pigeons in each group. 
native stimuli appeared immediately after pigeons pecked the stimulus 20 times. On horizontal line sample trials, a 6-sec delay followed the initial stimulus and was followed by the other pair of discriminative stimuli. As with the unsignaled group, one peck to the $\mathrm{S}+$ resulted in reinforcement, and the discriminative stimuli were counterbalanced in such a way that each hue served equally often as the $\mathrm{S}+$ and the $\mathrm{S}-$ and was equally often preceded by a delay or by no delay. The design for the signaled group is presented in the bottom panel of Figure 1.

All of the pigeons were trained until they reached a criterion of $90 \%$ correct on both discriminations for 4 out of 5 consecutive training sessions. They were then given a test session, followed by 10 additional training sessions; a 2 nd test session, followed by 10 additional training sessions; and finally a 3rd test session. Three test sessions were conducted to determine if stimulus preferences developed as a function of extended training.

Testing. Each test session involved 48 test trials. Test trials consisted of choice trials either between the two $\mathrm{S}+$ stimuli from training or between the two $\mathrm{S}-$ stimuli from training. On 16 test trials, 20 pecks to the initial stimulus (a white stimulus for pigeons in the unsignaled group or vertical lines for pigeons in the signaled group, as shown in the top panels of Figures 2 and 3) were followed immediately by the test stimuli. On another 16 test trials, 20 pecks to the initial stimulus (a white stimulus for pigeons in the unsignaled group or horizontal lines for pigeons in the signaled group, shown in the bottom panels of Figures 2 and 3 ) were followed by the test stimuli after a 6-sec delay. On the remaining 16 test trials, the test stimuli appeared at the start of the trial (i.e., there was no initial stimulus). An equal number of $\mathrm{S}+$ and $\mathrm{S}-$ test trials ( 8 each) appeared in each of the three initial-stimulus conditions. The initial stimuli were the same as those used during training of the two groups, and for the signaled group they predicted a delay or no delay as in training. On test trials, choice of either discriminative stimulus was reinforced on $50 \%$ of the trials. The dependent measure in each test session was the percentage choice of the positive and the negative stimuli that in training followed the delay.

\section{RESULTS}

The mean session-to-criterion scores for the signaled group (8.2 sessions, $S D=1.7$ ) were not significantly different from those of the unsignaled group (7.1 sessions, $S D=1.7)[t(14)=1.78, p>.05]$. However, for the signaled group it took significantly longer for the pigeons to acquire the simultaneous discrimination that followed a delay (mean sessions-to-criterion $=7.75$ ), than to acquire the one that followed no delay (mean sessionsto-criterion $=6.50)[t(7)=7.64, p<.05]$. It also took longer for the pigeons in the unsignaled group to acquire the simultaneous discrimination that followed a delay
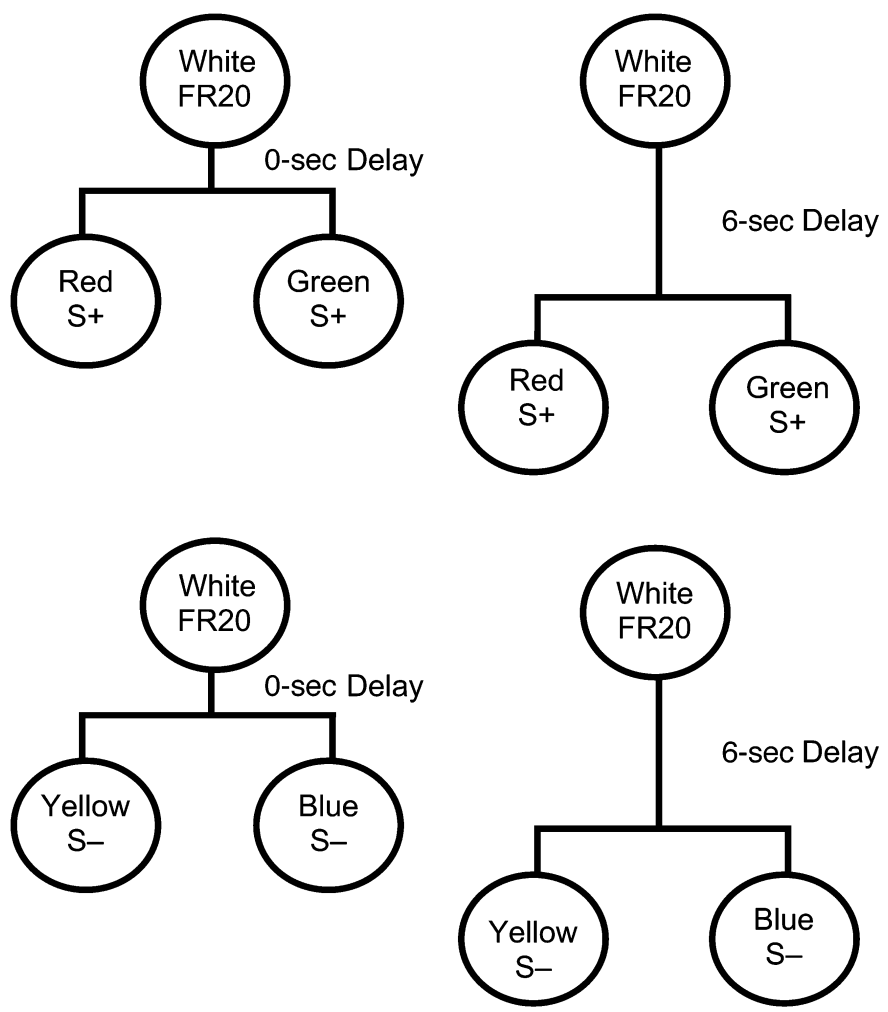

Figure 2. Sequence of events experienced by pigeons in the unsignaled group during testing. On one third of the test trials, the pigeons experienced the white initial stimulus and no delay followed by either the two $S+$ or the two $S-$ stimuli from training (left panel). On one third of the test trials, the pigeons experienced the white initial stimulus and the 6-sec delay followed by either the $S+$ or the $S-$ stimuli from training (right panel). Not shown are the remaining test trials, which began with the presentation of either the $S+$ or the $S-$ stimuli from training. 

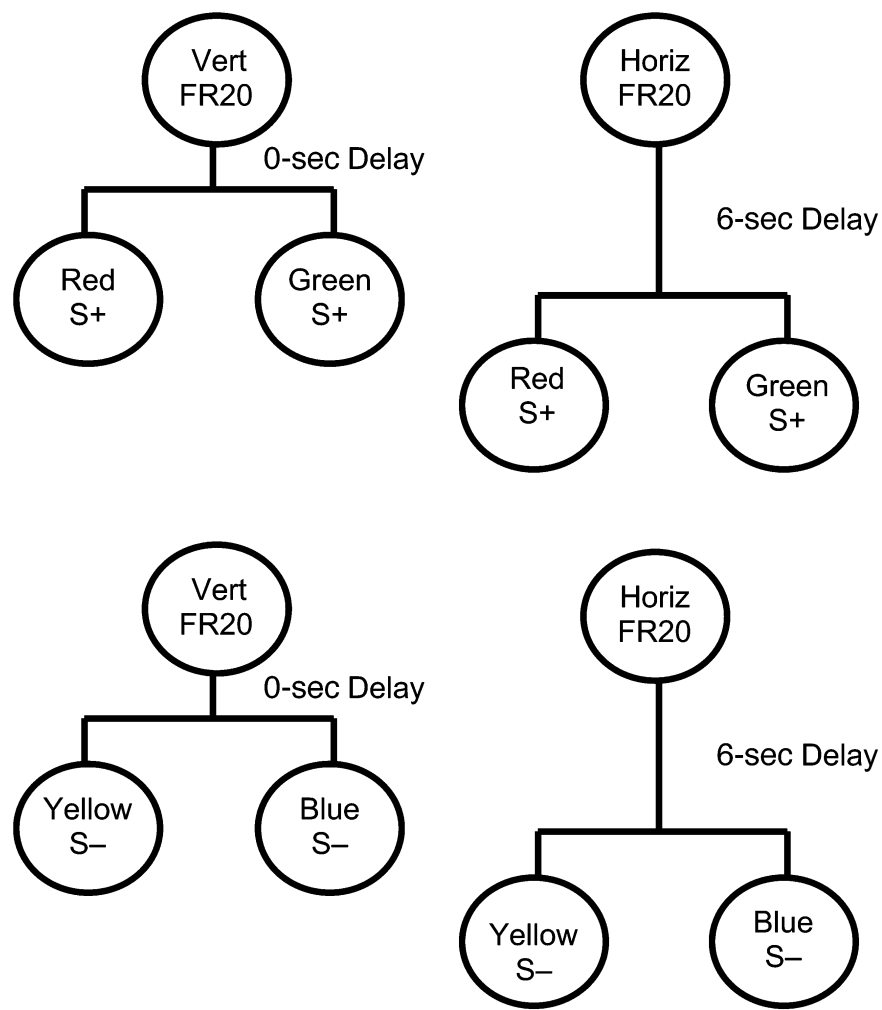

Figure 3. Sequence of events experienced by pigeons in the signaled group during testing. On one third of the test trials, the pigeons experienced the vertical line and no delay followed by either the two $S+$ or the two $S$ - stimuli from training (left panel). On one third of the test trials, the pigeons experienced the horizontal line and the 6-sec delay followed by either the $S+$ or the $S-$ stimuli from training (right panel). Not shown are the remaining test trials, which began with the presentation of either the $S+$ or the $S-$ stimuli from training.

(mean sessions-to-criterion $=7.00$ ) than to acquire the one that followed no delay (mean sessions-to-criterion $=$ $6.50)$, but in this case the difference was not statistically significant $[t(7)=1.32, p>.05]$.

The time to complete the fixed ratio (FR) requirement (time between sample onset and offset) on each kind of trial was assessed for the signaled group during each session. The times between sample onset and offset for each type of trial during the two training sessions before each test session were averaged and compared. Pigeons completed the pecking requirement in a mean of $16.49 \mathrm{sec}$ $(S D=6.29)$ on delay trials and in a mean of $7.43 \mathrm{sec}$ $(S D=4.31)$ on no-delay trials. These values were significantly different from each other $[t(7)=7.64, p<.05]$.

Choice of the positive stimulus that followed the delay, for pigeons in the signaled group, was quite similar for the three test sessions: $66.7 \%(S D=9.7), 61.5 \%(S D=$ $14.6)$, and $70.8 \%(S D=11.1)$ for Test Sessions 1, 2, and 3 , respectively. Similarly, for pigeons in the unsignaled group, choice of the positive stimulus that followed the delay was also quite similar for the three test sessions: $50.5 \%(S D=20.7), 49.0 \%(S D=11.5)$, and $41.7 \%$
$(S D=7.7)$ for Test Sessions 1, 2, and 3, respectively. A two-way mixed-effect analysis of variance (ANOVA) performed on the positive-stimulus choice data from the three test sessions, with group and test session as factors, indicated that there was a significant effect of group $[F(1,14)=16.78, p<.05]$ but no significant effect of test session $[F(2,28)=0.37, p>.05]$ and no significant group $\times$ test session interaction $[F(2,28)=2.36, p>$ $.05]$.

Choice of the negative stimulus that followed the delay, for pigeons in the signaled group, was also quite similar for the three test sessions- $56.2 \%(S D=18.9), 53.1 \%$ $(S D=15.1)$, and $58.3 \%(S D=14.3)$ for Test Sessions 1,2 , and 3, respectively - as well as for pigeons in the unsignaled group - 45.8\% $(S D=25.5), 46.4 \%(S D=$ $32.5)$, and $42.2 \%(S D=29.5)$, respectively. Another two-way mixed-effect ANOVA performed on the negativestimulus choice data from the three test sessions, with group and test session as factors, indicated that there was no significant effect of group $[F(1,14)=1.08, p>.05]$ or of test session $[F(2,28)=0.02]$ and no group $\times$ test session interaction $[F(2,28)=0.21]$. For this reason, all 
further analyses were performed with the data pooled over test sessions.

On test sessions, pigeons in the signaled group showed a significant preference $(65.4 \%, S D=9.0)$ for the positive stimulus that in training followed the delay over the positive stimulus that in training followed no delay $[t(7)=$ $4.85, p<.05]$. For pigeons in the unsignaled group, however, no significant preference between the two $\mathrm{S}+$ stimuli was found $(46.2 \%, S D=13.4)[t(7)<1]$. Neither the signaled nor the unsignaled group showed a significant preference for the $\mathrm{S}-$ stimulus that followed the delay in training $(55.9 \%, S D=15.9$, and $45.7 \%, S D=29.5$, respectively) $[t(7)=1.04, p<1$ and $t(7)<1$, respectively].

The test trial data were also analyzed for the effect of the initial stimulus (in test trials) on the preference for the discriminative stimuli. Initial stimulus type refers to the event that preceded the discriminative stimuli on test trials. On signaled test trials the initial stimulus was followed by the event that followed it in training-delay or no delay for horizontal or vertical lines, respectively. For the signaled group, a repeated measures ANOVA was conducted with the initial stimulus type (vertical, horizontal, no sample) as the independent variable. The means for each stimulus type for each group are presented in Table 1. The analysis indicated that choice of the positive stimulus that followed the delay did not differ significantly as a function of initial stimulus type $[F(2,21)=$ $2.51, p>.05]$.

A similar analysis performed on the data from the unsignaled group indicated that there was a significant effect of initial stimulus type $[F(2,21)=5.87, p<.05]$. A post hoc Scheffé analysis revealed that in test, pigeons in the unsignaled group chose the positive stimulus from delay trials in training significantly fewer times on nodelay trials $(27.13 \%, S D=25.8)$ than on delay trials $(60.38 \%, S D=18.5)(p<.05)$. In test, pigeons in the unsignaled group also chose the positive stimulus from delay trials in training significantly fewer times on nodelay trials than on no-stimulus trials $(51.0 \%, S D=$ 13.6) $(p<.05)$.

The effect of initial stimulus type on negative-stimulus test trials was examined also using a repeated measures ANOVA. The differences in preference for the negative

Table 1

Testing: Mean Percentage Choice of the Positive and Negative Stimuli That Followed the Delay During Training for the Signaled and Unsignaled Groups, Reported for Trials With an Initial Stimulus Followed by No Delay or a Delay and for Trials With No Initial Stimulus

\begin{tabular}{cccc}
\hline & \multicolumn{3}{c}{ Initial Stimulus } \\
\cline { 2 - 4 } Group & No Delay & Delay & No Stimulus \\
\hline Signaled & & & \\
Positive & 59.9 & 73.4 & 63.0 \\
Negative & 54.6 & 54.1 & 60.4 \\
Unsignaled & & & \\
Positive & 27.1 & 60.4 & 51.0 \\
Negative & 47.9 & 43.2 & 45.8 \\
\hline
\end{tabular}

stimuli among initial stimulus types were not statistically significant for either the signaled group $[F(2,21)=.278$, $p>.05]$ or the unsignaled group $[F(2,21)=.049, p>.05]$.

\section{DISCUSSION}

In agreement with the contrast hypothesis, pigeons in the signaled group chose the positive stimulus that followed the delay over the positive stimulus that followed no delay. These results support and extend Clement et al.'s (2000) finding that when a relatively more aversive event consistently precedes a stimulus that signals reinforcement, the value of that stimulus increases. The present experiment extended those findings to demonstrate that a delay can serve as the relatively aversive event.

Clement et al. (2000) interpreted their results in terms of the differential effort required to obtain the discriminative stimuli. However, requiring 20 pecks rather than 1 peck also increased the time to obtain the discriminative stimuli. Thus, effort and time were confounded in their study. The results of the present study suggest that delay alone is sufficient to produce a preference for the $\mathrm{S}+$ that follows greater effort, and it appears that differential effort is not required. Thus, although it is clear that pigeons would not choose to experience the trials that involve an added delay to reinforcement, it appears that the discriminative stimulus that follows acquires greater value.

Further research will determine whether differential effort by itself contributes to this preference. One should be able to determine the effect of differential effort by holding the delay constant and manipulating effort rather than holding effort constant and manipulating the delay.

The results of the present experiment also suggest that if discriminative stimuli are to increase in value, a delay must be predictable, because only pigeons in the signaled group showed a significant stimulus preference. It should be noted that in the Clement et al. (2000) study, in which differential pecking was required, no differential initial stimulus signaled the differential effort. In that study, however, the high-effort trials were signaled in a sense after the first peck; when the discriminative stimuli did not appear after one peck, the pigeons were predictably required to make 19 additional pecks to obtain the discriminative stimuli.

Signaling the delay also results in a significant increase in the duration of the trial. Pigeons took an average of $9.06 \mathrm{sec}$ longer to complete the FR20 requirement on signaled delay trials than on signaled no-delay trials. This means that signaled delay trials were generally about $15 \mathrm{sec}$ longer than signaled no-delay trials, whereas unsignaled delay trials were only $6 \mathrm{sec}$ longer than unsignaled no-delay trials. Thus, it may be that the greater difference in trial duration between delay and no-delay trials for the signaled group produced the stimulus preferences found.

It is also possible that the signal that predicted the delay for the signaled delay group provided an additional 
discriminative stimulus that may have facilitated acquisition of the simultaneous discriminations that followed. However, the signaled group did not acquire their simultaneous discriminations faster than the unsignaled group. More important, the simultaneous discrimination following the signaled delay was not acquired faster than the simultaneous discrimination following the signaled no-delay, and in fact it was acquired significantly more slowly. Perhaps the discrimination following the signaled delay was acquired more slowly than the discrimination following the signaled no-delay because the time of presentation of the discriminative stimuli following a delay was less certain than that following no delay. Alternatively, if the pigeons were using the initial stimulus as a means of predicting which discriminative stimuli would be presented, one would expect a greater loss of memory for the initial stimulus following a delay. In any case, slower acquisition of discrimination following a delay cannot account for the preference for the $\mathrm{S}+$ stimulus that followed the delay over that following no delay.

An alternative account of the data from the present experiment as well as those from previous studies (Clement et al., 2000; Clement \& Zentall, 2002) is based on the differential relative proximity of the signal for reinforcement to the reinforcement itself (i.e., the delay reduction hypothesis; Fantino, 1969). Although the actual delay to reinforcement signaled by the onset of the discriminative stimuli was the same for both $\mathrm{S}+$ stimuli (i.e., no delay), the proportion of the trial represented by the $\mathrm{S}+$ on delay trials was less than the proportion of the trial represented by the $\mathrm{S}+$ on no-delay trials. Thus, in a relative sense, the $\mathrm{S}+$ on a delay trial was closer to reinforcement, and in this sense the delay-preceded $\mathrm{S}+$ could have been considered to be a better predictor of reinforcement.

The relative delay reduction hypothesis is not unlike the contrast account suggested here in the sense that both involve a relational effect. In the case of the contrast account, the key relation is that between the relatively more aversive delay and the signal for reinforcement, whereas in the relative delay reduction account it is the relation between the total duration of the trial and the duration of the $\mathrm{S}+$.

According to the relative delay reduction theory, the pigeons in the unsignaled delay group should have shown an unambiguous preference for the positive stimulus that followed the 6-sec delay, because those trials were longer than the unsignaled no-delay trials, and longer trials place the discriminative stimuli relatively closer to the reinforcement. On the other hand, the fact that a preference for the positive stimulus that followed the 6-sec delay did occur for the signaled delay group is consistent with relative delay reduction theory, because signaling the delay increased the difference in trial duration between delay and no-delay trials by over $150 \%$.

One finding that is clearly not consistent with relative delay reduction was reported by Clement and Zentall (2002). In this series of experiments, it was found that the expectation of effort can create a stimulus preference in the absence of differences in trial duration, because in their experiments the effort and the discriminative stimuli did not occur on the same trials.

Specifically, Clement and Zentall (2002) presented pigeons with trials involving vertical-line initial stimuli on which a response would produce (probabilistically) either a stimulus that required high effort (30 pecks) or a simple simultaneous discrimination. On other trials, the pigeons were presented with horizontal-line initial stimuli on which a response would produce either a stimulus that required low effort (one peck) or a different simultaneous discrimination. On test trials, when the pigeons were given a choice between the two $\mathrm{S}+$ stimuli, they preferred the $\mathrm{S}+$ stimulus that followed the signal for possible high effort (vertical line) over the $\mathrm{S}+$ stimulus that followed the signal for possible low effort (horizontal line). The results of these experiments confirm the importance of the expectation of a relatively aversive event as the source of contrast. In Clement and Zentall's experiments, only the anticipation of greater effort preceded the discriminative stimuli, because the differential effort and the discriminative stimuli occurred on different trials. In these experiments, trials involving discriminative stimuli were all of the same duration, and for this reason relative delay reduction theory should have difficulty accounting for the preference between the two positive stimuli. Thus, Clement and Zentall's results together with the present findings are more consistent with a contrast account.

In earlier research in which a contrast effect of the type reported in the present experiment was found (Clement et al., 2000; Clement \& Zentall, 2002), there was no evidence that the initial stimulus on test trials had any effect on the stimulus preferences that were found. In the present experiment, although there was no significant overall preference for either of the $\mathrm{S}+$ stimuli over the other, there was a significant difference in preference when the test trial was initiated by an initial stimulus and no delay, as compared with either an initial stimulus and a delay or no initial stimulus. More specifically, when a nodelay event preceded the $\mathrm{S}+$ choice, pigeons in the unsignaled group showed a greater preference for the $\mathrm{S}+$ that in training was preceded by the no-delay event. This result could be viewed as a context effect (or generalization decrement), because in training the absence of a delay consistently was followed by that particular $\mathrm{S}+$. This conclusion should be accepted with caution, however, because such an effect has not been found in earlier research or in the signaled group in the present experiment. On the other hand, such a context effect may appear only when there is little overall preference for the $\mathrm{S}+$ that follows the relatively more aversive event.

Lawrence and Festinger (1962) have proposed that cognitive dissonance theory- the theory that dissonance produced by a discrepancy between behavior (undergoing an unpleasant initiation) and beliefs (that one should 
avoid unpleasant experiences) - can account not only for much seemingly paradoxical human behavior but also for the behavior of nonhuman animals (e.g., contrast effects and resistance to extinction following partial reinforcement). We suggest here that relative change in hedonic state may provide a more parsimonious account of these phenomena when found not only in nonhuman animals but also perhaps in humans. The present findings also may have implications for cognitive dissonance research with humans. For example, using a design quite similar in principle to that used in the present research, Aronson and Mills (1959) invited female college students to join a discussion group. One group had to experience an embarrassing prescreening (an unpleasant episode), two others did not. After listening to a sample of their group's discussion (the same for all groups) they were asked to rate the discussion and the group members. Participants who had experienced the embarrassing prescreening judged both the discussion and the group members to be more interesting and more intelligent than did the control groups. Although these results were interpreted in terms of cognitive dissonance, the fact that a similar effect can be found in pigeons when a delay is substituted for the unpleasant episode suggests that it may be fruitful to ask if similar mechanisms are involved.

Whether contrast can account for effects that have been attributed to cognitive dissonance is not clear. Nevertheless, it may be important to determine the role played by contrast in existing manipulations that have been previously accounted for in terms of more complex cognitive processes such as cognitive dissonance.

\section{REFERENCES}

Aronson, E., \& MiLls, J. (1959). The effect of severity of initiation on liking for a group. Journal of Abnormal \& Social Psychology, 59, 177-181.

Bouton, M. E. (1993). Context, time, and memory retrieval in the interference paradigms of Pavlovian conditioning. Psychological Bulletin, 114, 80-99.

Clement, T. S., Feltus, J. R., Kaiser, D. H., \& Zentall, T. R. (2000). "Work ethic" in pigeons: Reward value is directly related to the effort or time required to obtain the reward. Psychonomic Bulletin \& Review, 7, 100-106.

Clement, T. S., \& Zentall, T. R. (2002). Second-order contrast based on the expectation of effort and reinforcement. Journal of Experimental Psychology: Animal Behavior Processes, 28, 64-74.

FANTINO, E. (1969). Choice and rate of reinforcement. Journal of the Experimental Analysis of Behavior, 12, 723-730.

Hull, C. L. (1943). Principles of behavior. New York: Appleton-CenturyCrofts.

LaWrence, D. H., \& Festinger, L. (1962). Deterrents and reinforcement: The psychology of insufficient reward. Stanford, CA: Stanford University Press.

Spetch, M. L., Wilkie, D. M., \& Pinel, J. P. J. (1981). Backward conditioning: A reevaluation of the empirical evidence. Psychological Bulletin, 89, 163-175.

(Manuscript received June 17, 2003

revision accepted for publication October 28, 2003.) 